\title{
Chitosan nanoparticles: Preparation, food applications and health benefits
}

\author{
Avtar Singh, Ajay Mittal, Soottawat Benjakul ${ }^{*}$ \\ International Center of Excellence in Seafood Science and Innovation (ICE-SSI), Faculty of Agro-Industry, \\ Prince of Songkla University, Songkhla 90110 Thailand
}

*Corresponding author, e-mail: soottawat.b@psu.ac.th

Received 18 Jan 2021

Accepted 28 Jan 2021

\begin{abstract}
Chitosan (CS) is a biodegradable and nontoxic natural polymer extensively employed in food and biomedical industries pertaining to its excellent bioactivities and biocompatibility. Superior gelling characteristic of CS, due to its polycationic nature, is achieved in the presence of anionic substances. The ionic gelation method with the aid of mechanized stirring or ultrasound has been popularly used to produce CS nanoparticles (CSNPs) of various sizes (84-600 nm) with encapsulation efficiency of 23-97\%. In addition, other techniques, including emulsion cross-linking, coacervation and reverse micelle have been implemented. Moreover, nanoparticles (NPs) of various sizes (70-1000 nm) have been produced by coating nanoliposome with the help of CS to enhance their stability and activities. The CSNPs loaded with active compounds have improved the stability during storage, sustained release, higher bioactivities such as antioxidant and antimicrobial activities than the free form. This review also aims to cover the major applications of various CSNPs as an encapsulating agent, food additives and edible coatings. Finally, the efficacy of CSNPs is also addressed for medical uses, such as reducing glucose or fat levels, wound healing and cancer treatments.
\end{abstract}

KEYWORDS: chitosan, ionic gelation, nanoparticles, edible coating, human health

\section{INTRODUCTION}

Chitosan (CS) is a deacetylated (DA) product of chitin, which is broadly used in medicinal and pharma industry due to its biodegradable, biocompatible and less toxic nature [1]. It also has a wide array of usages such as drug delivery, packaging material, antibacterial agent, tissue engineering, antiaging agent, antibody response enhancer as well as the remedy of ailments (such as cancer treatment) [2,3]. In general, CS is an aqueous acid soluble biomolecule. Low MW CSs (2.8-87.7 kDa) possessed higher solubility and functional activities than high MW counterparts (604-931 kDa) $[2,4]$. To better exploit CS, it has been reduced to low MW using various methods, such as hydrolysis by chemical, enzymatic and physical methods. The conjugation with various compounds to enhance the potential applications of CS has been documented [4]. Apart from various bioactivities, cationic nature of CS associated with the amino group at C-2 shows a potential to form gel or acts as a crosslinking agent with unique biophysical and biochemical properties. Those properties of CS in conjunction with the use of nanotechnology have led to the formation of CS nanoparticles (CSNPs).

Nanoparticles (NPs) possess unique properties such as color, diffusivity, toxicity, solubility, strength as well as magnetic, optical, and thermo-dynamic properties due to high surface ratio [5]. It can interact with atoms, molecules or macromolecules for the targeted reaction. CSNPs have both characteristics of CS and NPs, such as surface and interface effect, small size and quantum size effects. Applications of CSNPs as food preservative, active packaging material, drug carrier, and encapsulating agent for bioactive compounds have been gaining interest for the last few decades [6]. Therefore, CSNPs' preparation and their applications in various sectors are revisited in this review.

\section{PREPARATION OF CSNPS}

Various methods have been developed to formulate CS particulates as a delivery system. Those methods are mainly dependent upon the nature of the bioactive compounds, particle size, release kinetic, stability, residual toxicity, and mode of delivery of the final products [7]. In general, ionic gelation, emulsion cross-linking and coacervation or precipitation methods were widely employed in the preparation of CSNPs [7-9]. In addition, spray drying, emulsion-droplet coalescence, reverse micellar, and sieving are also known for the preparation of NPs, 
but are still rarely used $[7,10]$.

\section{Ionic gelation (IG)}

IG, also called ion-induced gelation, is a non-toxic, organic solvent-free, easy, and regulated method to produce the desired particles. For preparation of CSNPs via IG method, CS (0.1-2\%, w/v) solution is firstly added to the tripolyphosphate (TPP) solution with ranged concentrations $(0.1-0.5 \%, \mathrm{w} / \mathrm{v})$ via drop-wise process and constant stirring. Sizes and encapsulation efficiency (EE) of the CSNPs varied in the ranges of 84-600 $\mathrm{nm}$ or higher and 23-97\%, respectively (Fig. 1A and Table 1). The catechinloaded CSNPs prepared by IG method $(0.05$ or $0.1 \%$ of CS and $0.1 \%$ TPP) have the size range of 110 $130 \mathrm{~nm}$ and $\mathrm{EE}$ of $90 \%$ when CS/TPP ratio of $2: 1$ and pH 5.5 were used [11]. The positively charged amino groups of CS at the C-2 position interact with negatively charged phosphoric groups of TPP [12]. During this process, three kinds of phenomena occur, involving (1) solution, (2) aggregation and (3) opalescent suspension, in which the last one determines the formation of NPs [13]. Various factors, such as concentration, MW and ratios of ingredients used, mixing style, and $\mathrm{pH}$ of the system, have the influence on the formation of NPs. Generally, with increasing MW of CS, the particle size of NPs was increased, which was more likely due to the formation of long chain beaded structure of NP prepared using IG method [14]. Similarly, EE was influenced by the MW of CS. For example, when MW of CS $(1.5 \mathrm{mg} / \mathrm{ml})$ was increased from 50 to $150 \mathrm{kDa}$, the EE of tea catechin augmented from 26 to $47 \%$, respectively, and then decreased to $36 \%$, when MW was increased further to $300 \mathrm{kDa}$ [15]. The addition rate of TPP modified the shape and chain length of NPs beads. This was more likely due to competition between phosphate ion and hydroxyl group to interact with the amino group of CS. Various bioactive compounds, including polyphenols, have been encapsulated in CSNPs prepared by IG methods (Table 1). Particles produced via this method possessed high dispersibility index but showed non-uniform surface morphology, lack of active surface site for attachment as well as poor mechanical properties $[13,14]$.

\section{Liposomes}

Liposomes are colloidal particulates with lipid bilayer membranous structure (Fig. 1B). Liposomes can be used in both lipid and aqueous phases as a delivery agent for various pharma drug; and they have also been used as vector for various macromolecules, such as proteins, DNA and RNA $[16,17]$. Generally, nanoliposomes (NLs) formation is based on hydrophilic and hydrophobic interaction between phospholipids and water molecules. The sizes of NLs are varied widely $(70-600 \mathrm{~nm})$, based on their applications [18]. Various methods, such as thin film evaporation, sonication, reverse phase evaporation, melting, and freezing-thawing, have been employed to obtain different types of liposomes, multilamellar, large or small unilamellar, etc [16]. The different structures of NLs were governed by the nature and concentration of core material, phospholipids and stabilizers. Those factors affect size, EE and release of the active compounds [19]. The cationic nature of CS forms an ionic bond with negatively charged phospholipids, leading to the formation of CS-coated NLs. Quercetin was loaded into CS-lecithin NLs (Q-NPs) through the electrodeposition method [20]. The QNPs possessed smaller particle size $(350-600 \mathrm{~nm})$ and high EE (71.14\%). The zeta potential of QNPs was $35.12 \mathrm{mV}$, suggesting that vesicle prepared using this method had high stability. In general, zeta potential greater than $30 \mathrm{mV}$ or less than $-30 \mathrm{mV}$ can be dispersed stably in medium [20]. In another study, when vitamin-C-NLs were prepared using ethanol as a solvent along with phosphatidylcholine and cholesterol ratios of 40:60 and 60:40, NLs having sizes of 97.4 and $95.8 \mathrm{~nm}$ were produced, respectively. When those NLs were coated with CS at various concentrations $(0.1-1.0 \%)$, mean particle diameter was increased (100-460 nm) with increasing CS concentrations [21]. This was more likely associated with the augmenting number of layers or interactions of CS chains over the NLs resulting in a higher viscosity of the suspension.

\section{Emulsification method}

The emulsification process is implemented to produce both nano- and micro-particles using waterin-oil or oil-in-water nanoemulsion (NE) with and without a cross-linking agent such as glutaraldehyde or TPP $[22,23]$. The cross-linking agents induced hardening of the droplets formed during emulsification [24]. CSNPs, having an average diameter of $250 \mathrm{~nm}$, were used for encapsulation of 5-fluorouracil (5FU) or immobilization of aminopentyl-carbamoyl-5FU or aminopentyl-estermethylene-5FU in the presence of glutaraldehyde using emulsion method. In this method, $10 \mathrm{ml}$ of glutaraldehyde was saturated with toluene $(11 \mathrm{mg} / \mathrm{ml})$ containing $10 \%(\mathrm{v} / \mathrm{v})$ Span 80 [25]. Generally, the water-in-oil NE is prepared by in- 
A

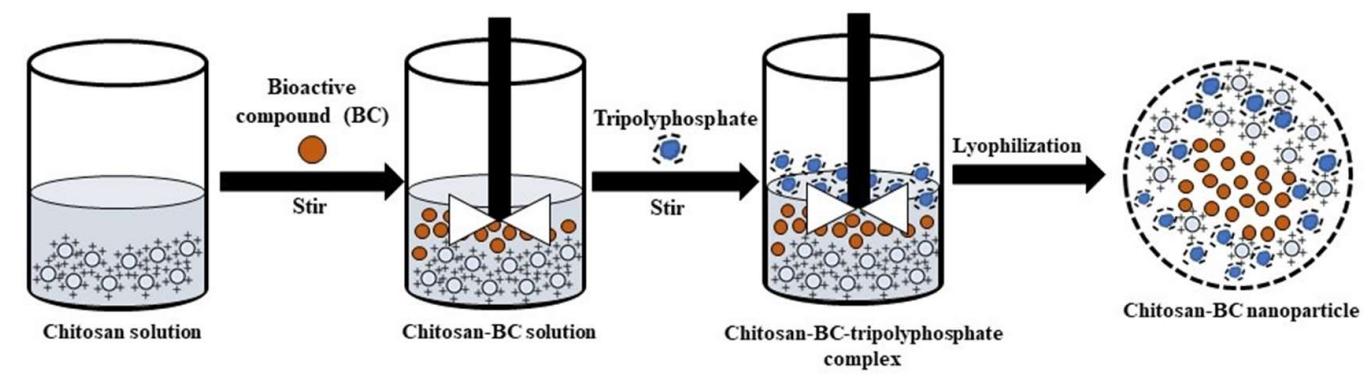

B
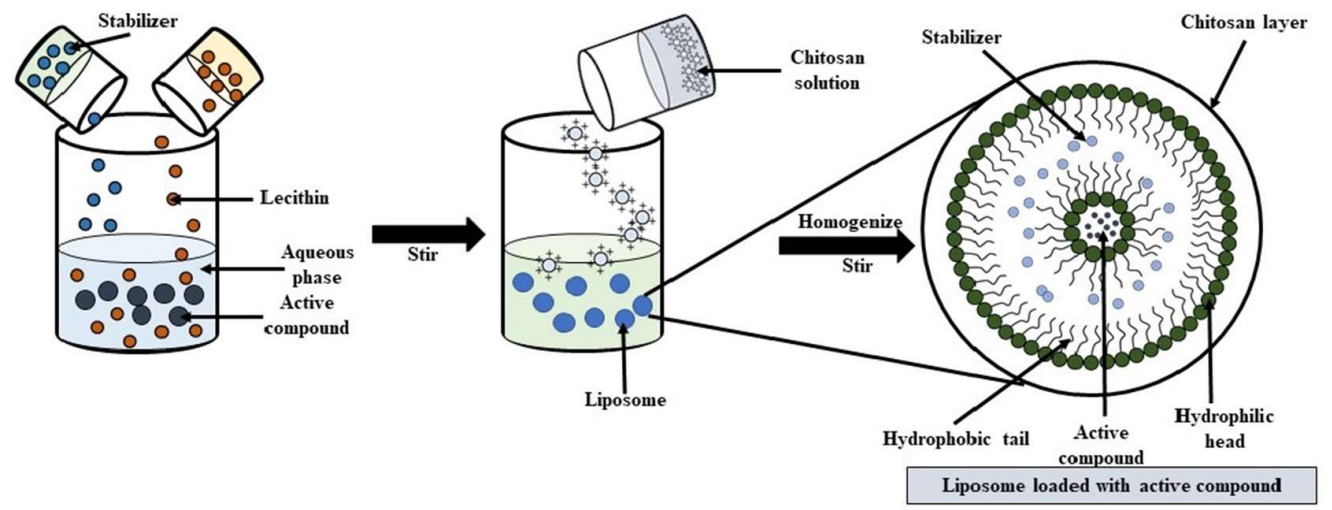

Fig. 1 Preparation of chitosan nanoparticles loaded with active compounds through ionic gelation (A) and liposome (B) methods.

Table 1 Characteristics and applications of chitosan nanoparticles loaded with active compounds prepared using various methods.

\begin{tabular}{|c|c|c|c|c|c|c|}
\hline Shell & Method & Core & $\mathrm{EE}(\%)$ & Size (nm) & Activity/application & Ref. \\
\hline \multirow{15}{*}{ Chitosan } & $\begin{array}{l}\text { Oil-in-water } \\
\text { emulsion and } \\
\text { ionic gelation }\end{array}$ & Carvacrol & $14-81$ & $40-80$ & $\begin{array}{l}\text { Antimicrobial activity against } S \text {. aureus, } B \text {. cereus and } \\
\text { E. coli }\end{array}$ & [62] \\
\hline & Ionic gelation & $\begin{array}{l}\text { Elsholtzia splendens } \\
\text { extract }\end{array}$ & $23-31$ & $350-520$ & Antioxidant and lipid peroxidation inhibitory activity & [8] \\
\hline & Ionic gelation & Polyphenols & $59-90$ & $300-600$ & Antimicrobial activity & [9] \\
\hline & Ionic gelation & Catechins & 90 & 130 & $\begin{array}{l}\text { Enhance muco-adhesivity and controlled release of cat- } \\
\text { echin in GIT }\end{array}$ & [11] \\
\hline & Ionic gelation & Curcumin & 75 & 160 & Antimicrobial activity against $S$. aureus and $P$ aeruginosa & [63] \\
\hline & Ionic gelation & Ellagic acid & 94 & 176 & Enhanced cytotoxicity in human oral cancer cell line & [64] \\
\hline & Ionic gelation & Gallic acid & & 453 & Antimicrobial activity & [65] \\
\hline & Ionic gelation & $\begin{array}{l}\text { Green tea catechins } \\
\text { and EGCG }\end{array}$ & & $432-440$ & Increase intestinal absorption & [66] \\
\hline & Ionic gelation & Oxaliplatin & 89 & 103 & Mucoadhesive topical treatment of oral tumors & [67] \\
\hline & Self-assembly & Catechins & 74 & $130-150$ & $\begin{array}{l}\text { Enhance the transport of tea catechins across Caco- } 2 \text { cell } \\
\text { monolayers }\end{array}$ & [43] \\
\hline & Coprecipitation & Doxorubicin & $99-81$ & $3-8$ & Anticancer & [68] \\
\hline & $\begin{array}{l}\text { Emulsion- } \\
\text { ionic gelation }\end{array}$ & Clove essential oil & 46 & 100 & $\begin{array}{l}\text { Enhanced antifungal property of CEO due to controlled } \\
\text { release }\end{array}$ & [37] \\
\hline & Coacervation & $\begin{array}{l}\text { Iron casein succiny- } \\
\text { late }\end{array}$ & & 830-1070 & Enhanced delivery of iron in Caco-2 cell model & [31] \\
\hline & Electrospray & Tea polyphenols & 66.7 & 205 & Incorporated in gelatin film & [44] \\
\hline & Liposome & Quercetin & 71 & $350-600$ & $\begin{array}{l}\text { Enhanced inhibitory effect of nanoliposome on HepG2 } \\
\text { cells }\end{array}$ & [37] \\
\hline \multirow[t]{2}{*}{$\beta$-chitosan } & Ionic gelation & $\begin{array}{l}\text { Catechins and } \\
\text { catechins-Zn }\end{array}$ & $50-84$ & $208-590$ & Antibacterial activity against $L$. innocua and $E$. coli & [41] \\
\hline & Ionic gelation & Tea polyphenols-Zn & 97 & 84 & Antioxidant activity & [69] \\
\hline
\end{tabular}


jecting an organic phase loaded with an emulsifier containing oil, into a mixture of bioactive compound and CS with the aid of mechanical stirring (Fig. 2A) [26]. The oil-in-water NE is composed of minute lipid droplets containing lipophilic active compounds, which were dispersed in an aqueous medium. In both water-in-oil and oil-in-water NEs, the final size of the particles mainly depends on the cross-linking agent and stirring speed [24]. The cross-linked agents undergo reaction with CS via amino groups to form an emulsion followed by the formation of nano- or micro particles [24]. Moreover, CS can be used to coat the emulsion, via electrostatic adsorption, without a cross-linking agent (chitosomes) [27]. However, the emulsification method is tedious and requires toxic crosslinking agents, thus limiting the use of this method for preparation of NPs.

\section{Other methods}

Besides the aforementioned methods, CSNPs have been prepared by simple and complex coacervation, reverse micelles, self-assembly, and spray drying methods $[7,10,26]$. In general, a homogeneous solution of charged CS and cross-linking agents underwent liquid to liquid phase separation, which results in the formation of polymer-rich dense and transparent phase (Fig. 2B) [28]. They are of mainly two types, simple and complex coacervations. For simple coacervation, the electrostatic forces occur between opposite-charge molecules of a single biopolymer, whereas the complex coacervation is made by a complex set of electrostatic forces between negative-charge biopolymers (e.g., gum Arabic) and positive-charge biopolymers (e.g., chitosan) [29, 30]. For CS, Min, Cho [31] produced CSNPs from CS (degree of deacetylation $<90 \%$ ) for immobilizing iron casein succinylate (ICS) using the complex coacervation method. The mean diameter (830-1070 nm) of CS-ICS NPs was increased with augmenting concentrations of both biomolecules. Overall, these formulations have substantial potential to deliver various bioactive compounds, not only for drug delivery but also for food systems $[7,26]$. For the reverse micelles technique, surfactant dissolved in organic solvent was mixed with CSbioactive compound solution and followed by constant stirring [22]. Thereafter, the mixture was centrifuged or sonicated to obtain the desired particle size [7]. The schematic diagram of the reverse micelles process is given in Fig. 2C. For self-assembly, the organization of new structures from initial components including atoms, molecules, or NPs mostly undergo non-covalent interactions [32,33]. Therefore, the process can be simply affected by $\mathrm{pH}$, temperature, and mechanical stresses. In spray drying method, the sample solution is vaporized in a hot-air drying chamber, in which the NPs can be obtained as dried powder within an electrostatic collector [34]. The major limitation of the method is associated with viscosity of biopolymer suspension, which may block the vaporizer nozzle and resulted in lower the yield [35]. Owing to the difficulty in operation or chemicals used, these methods have been implemented rarely $[7,26]$.

Overall, CSNPs have been served as a vector for various drugs and bioactive compounds associated with their capability of stabilization and release of active core compounds.

\section{APPLICATIONS OF CSNPS}

\section{Encapsulating agent}

Generally, labile compounds, such as tea polyphenols, essential oils, vitamins, enzymes, etc., are unstable and can be altered or lose their bioactivities during the formulation, food processing, digestion, and delivery. Therefore, to tackle this problem, encapsulation technology has been introduced widely, in which CSNPs are one of the major encapsulating agents. For example, CSNPs prepared by the emulsion-electrostatic interaction method with the help of TPP reduced the formation of hydroperoxide formation in krill oil stored for two weeks at $45^{\circ} \mathrm{C}$ [36]. Similarly, clove essentialoil (CEO) encapsulated into CSNPs by emulsionIG method showed lower degradation of volatile compounds as well as improved in vitro antifungal activity due to the controlled release of CEO [37]. Furthermore, Hadidi, Pouramin [38] observed the enhanced antioxidant potential of entrapped CEO. Higher inhibition of Staphylococcus aureus and Listeria monocytogenes was also achieved by entrapped CEO when compared with free CEO. The higher antimicrobial activity was more likely due to the round shape of NP, in which polycationic CSNPs can interact with the negatively-charged surface of bacteria to a higher extent. Since the large surface area of CSNPs can absorb onto the bacterial surface and cause the leakage of intracellular compounds and subsequent cell death [39]. For smaller particles, penetration into cytosol of bacterial cell can cause failure of metabolic pathways of bacteria [4]. The particle size of NPs has been known to influence its antimicrobial activity, smaller particles possessing higher activity [40]. The minimum inhibitory 
A
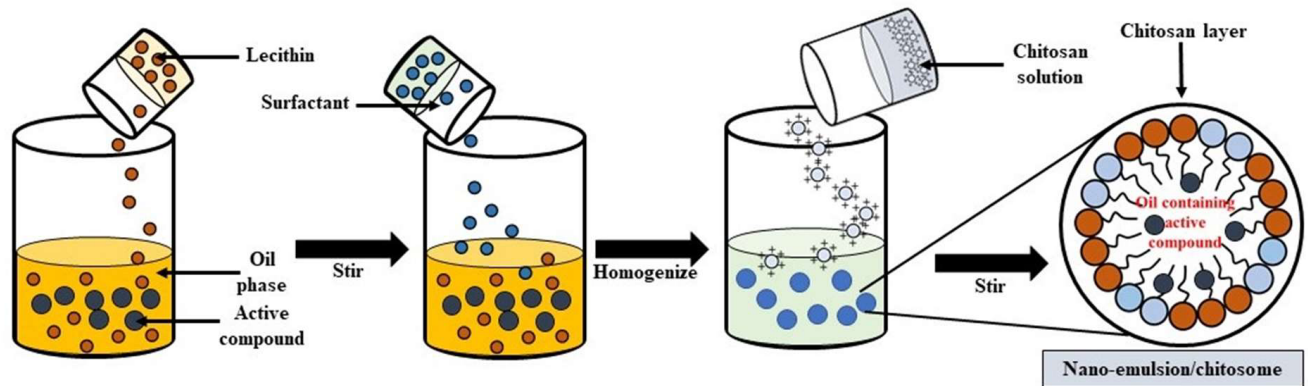

B

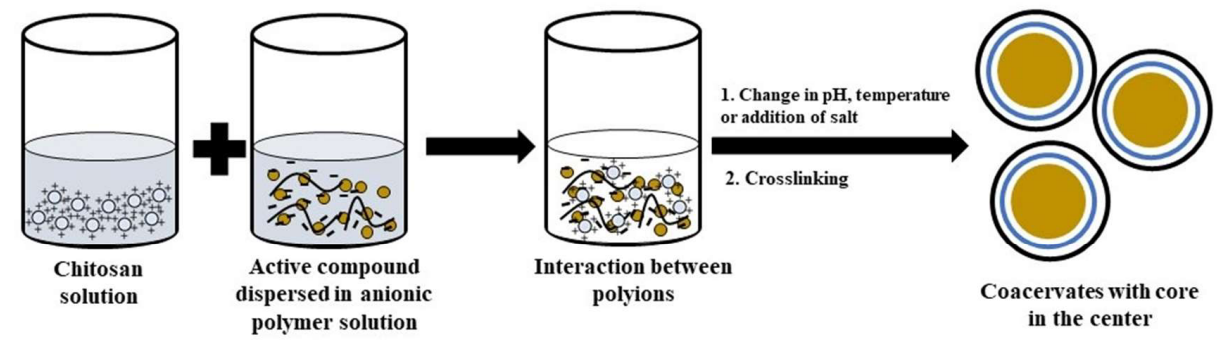

$\mathrm{C}$

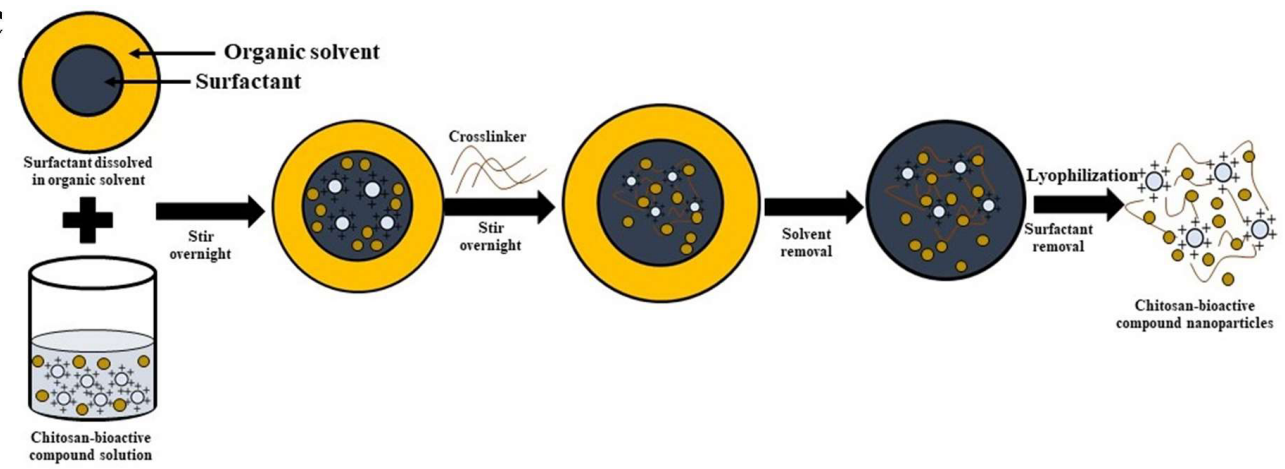

Fig. 2 Preparation of chitosan nanoparticles loaded with active compounds through nano-emulsion (A), coacervation (B), and reverse micelle (C) methods.

concentration (MIC) and minimum bactericidal concentration (MBC) values of the smallest particle size (208 nm) of catechins-Zn complex encapsulated by $\beta$-CSNPs against Listeria innocua and Escherichia coli were 0.03 and $0.06 \mathrm{mg} / \mathrm{ml}$, and 0.06 and $0.13 \mathrm{mg} / \mathrm{ml}$, respectively [41]. Conversely when particle size was increased to $590 \mathrm{~nm}$, the MIC and $\mathrm{MBC}$ values of 0.13 and $0.03 \mathrm{mg} / \mathrm{ml}$ and 0.3 and $0.13 \mathrm{mg} / \mathrm{ml}$ against aforementioned bacteria were obtained. It was noted that MIC and MBC values were increased against both bacteria, indicating lower antibacterial activity. However, MIC for $L$. innocua was not changed with increasing size. This might be associated with lower ability of larger particles to penetrate through the bacteria cell wall, thus lowering its antimicrobial effect. In addition, CS possesses an excellent antimicrobial activity, which synergistically enhances the potential effect of CSNPs [2-4]. The samples encapsulated using NLs had been determined for various activities. For example, Q-NLs showed improved stability and higher antioxidant activity compared with free quercetin, which might be due to the enhanced solubility of quercetin NLs [20]. Vitamin-C NLs coated with CS and stored at $4{ }^{\circ} \mathrm{C}$ for 15 weeks, could maintain its antioxidant activity indicating higher stability of the coated samples in comparison to the free form [21]. Additionally, CSNLs has been used as a carrier for several drugs. Docetaxel, a popular anticancer drug, was loaded into NLs, which was further encapsulated inside folate grafted thiolatedCS. It effectively inhibited MD-MB-231 breast cancer cells, showing $\mathrm{IC}_{50}$ value of 200-times lower than that of pure docetaxel [42]. This indicated the 


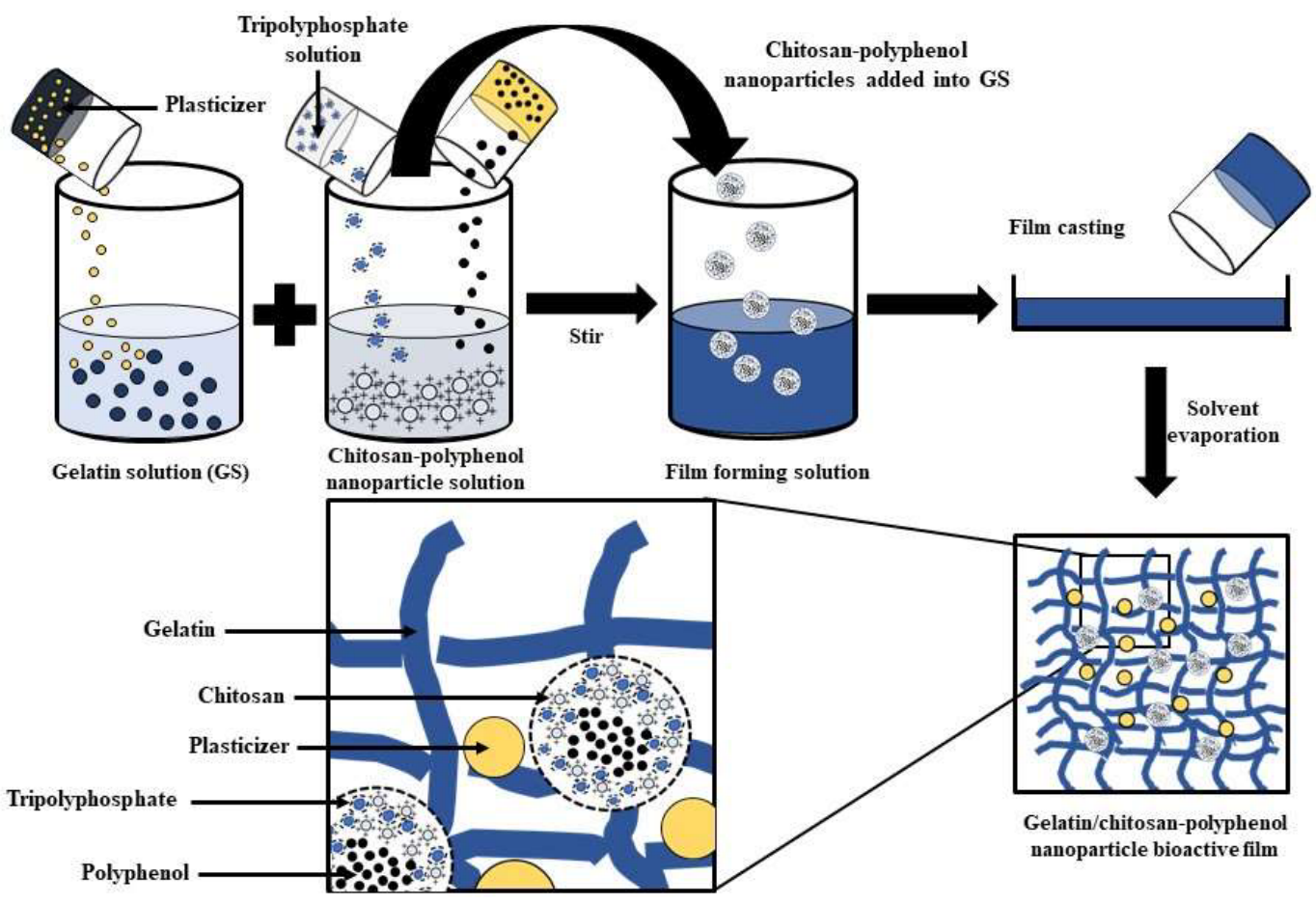

Fig. 3 Preparation of gelatin film incorporated with chitosan-polyphenol nanoparticles.

enhanced oral bioavailability, which was confirmed by pharmacokinetics study. Similar observations were obtained for berberine hydrochloride when loaded into CS coated NLs [18].

Oral administration of sensitive drugs is limited by the harsh conditions of the gastrointestinal tract (GIT). Therefore, self-assembled NPs, prepared by $\mathrm{CS}$ and an edible polypeptide, were used to deliver the tea catechins into GIT [43]. The increase in paracellular transport of tea catechins was due to positive surface charge of NPs, which opened tight junctions between Caco-2 cells. Similarly, CS and sodium TPP was used for synthesis of catechinloaded CSNPs with the size of 110-130 nm and possessing the controlled release of catechin in GIT [11].

\section{Edible coating or film}

The shelf-life of a wide array of foods has been increased by the application of CS and its derivatives [26]. Hence, the ability of CSNPs to control the release of various bioactive components can enhance the antimicrobial/antioxidant properties of food packaging materials. The gelatin film incorporated with tea polyphenol loaded CSNPs was generated through electrospray (Fig. 3) [44]. Beluga (Huso huso) fish fillets treated with an ed- ible coating containing CSNPs loaded with fennel essential oils in combination with modified atmospheric packaging showed lower value of peroxide, total volatile nitrogen and thiobarbituric acid [45]. Also, lower mesophilic, psychotropic, Pseudomonas spp., and lactic acid bacteria counts were noted in fillets coated with CSNPs as compared to those without CSNPs [45]. Similarly, the shelf-life of Litopenaeus vannamei (whiteleg shrimp) stored at $4{ }^{\circ} \mathrm{C}$ was increased up to 10 days, when coated with CSNPs [46]. The retarded lipid oxidation of silver carp (Hypophthalmicthys molitrix) fillets was observed up to 12 days at $4^{\circ} \mathrm{C}$ when coated with CSNPs in combination with orange or pomegranate peel extract [47]. However, CSNPs of pomegranate peel extract was more effective than orange peel extract. In another study, Alboghbeish and Khodanazary [48] compared quality characteristics of Carangoides coeruleopinnatus fillets coated with CS and CSNPs for 12 days at $4{ }^{\circ} \mathrm{C}$. NPs coating on fillets retarded lipid oxidation, as well as growth of microorganisms, more efficiently than CS coated fillets. Recently, the lowest peroxide value of soybean oil on day 14 was documented when packaged in gelatin film incorporated with $30 \%(\mathrm{w} / \mathrm{v})$ tea polyphenol-loaded CSNPs [44].

Despite the shelf-life extension of various foods, 
CSNPs also improved the mechanical and physicochemical properties of films. Tara gum films were developed using the incorporation of bulk CS and CSNPs. Films added with CSNPs showed higher mechanical strength, antimicrobial activity, water solubility and lower hydrophilicity than CS added film [49]. In another study, solubility of CS$\mathrm{ZnO}-\mathrm{NPs}$ composite coating was increased by $42 \%$, whereas degree of swelling was reduced by $80 \%$ when compared to the CS coating [50]. Gelatin films have been developed for food packaging; but still their mechanical properties are low, and preservative effects are poor on various food products. Hosseini, Rezaei [51] developed fish gelatin films using CSNPs as a filler, which resulted in augmented mechanical properties and increased water vapor barrier by $50 \%$. Furthermore, the composite films based on gelatin/CSNPs containing oregano essential oil were more flexible, with decreased surface hydrophilicity and water vapor permeability. Thus, CSNPs can be potentially used in bio-composite films to enhance their mechanical properties and bioactivities.

\section{Other applications in foods}

CSNPs were prepared by IG process and used as a cryoprotectant in grey mullet surimi [52]. The incorporation of NPs in surimi efficiently impeded the microbial load and lipid oxidation by lowering the formation of thiobarbituric acid substances and conjugated dienes. Moreover, CSNPs were also able to inhibit oxidation of fish myoglobin via lowering transformation of heme iron. Besides, CSNPs were used as a glazing material for cryogenically frozen shrimp [53]. Glazing materials containing CSNPs could suppress lipid oxidation with no negative effects on color and texture of frozen shrimps during storage [53].

\section{Human health benefits}

CS plays an important role in maintaining stability and sustaining release of bioactive components. Moreover, its ability to act as antioxidant, antitumor, antiviral agent, etc. synergistically enhanced the efficacies of various drugs and therapeutic compounds. Various studies have been performed in vivo or in vitro conditions to elucidate the CS's properties.

\section{Antitumor activity}

CSNPs, in combination with various derivatives, have been used at a large scale in the pharma or medicinal industry as wound healing, anticancer, drug delivery agents, etc. CS-folate conjugated NPs loaded with EGCG (mean diameter of $400 \mathrm{~nm}$ ) showed higher in vitro antitumor activity against various cell lines, such as HeLa, H1299 and Capan-1 cells, than free EGCG [54]. The inhibitions of NPs towards HeLa, H1299 and Capan-1 cells were 85, 56 and 64\%, respectively. Similarly, CS-folate conjugated NPs encapsulated with catechin (average diameter of $255 \mathrm{~nm}$ ) showed antitumor activity against MCF-7 and HepG-2 cells [55]. In both cells, the loaded CSNPs showed a higher antiproliferation effect than the blank NPs. Moreover, MCF-7 cells were more sensitive to catechin-loaded folate-conjugated CSNPs than HepG-2 cells, indicating that the CSNPs had a higher cytotoxic effect on folate receptor-positive tumor cells. CSNPs possessed positive charges on their surface and high affinity toward negatively charged tumor cell membrane, thereby causing endocytosis via releasing active components into the cytosol. As a result, various cellular mechanisms were altered and ultimately led to cell death.

\section{Wound healing (WH) activity}

The natural origin and biocompatibility of CS promoted its use for WH because CS fuels haemostasis and expedites tissue renewal. The hydrophilic character and homogenous structure of CS, relative to glycosaminoglycans, serve as an attractive material for a tissue engineering scaffold [56]. CSNPs of average diameter $208.5 \mathrm{~nm}$ and positive zeta potential about $24.2 \mathrm{mV}$, prepared by IG method and loaded with calcium alginate hydrogel, exhibited remarkable antibacterial and pro-inflammatory activity, as indicated by the inhibition of generation of reactive oxygen species. Subsequently, the process promoted vascular endothelial cell invasion, metastasis and neovascularization, resulting in a higher rate of $\mathrm{WH}$. The rate of wound closure was increased to $85.6 \%$ within 7 days and $98.7 \%$ within 14 days [57]. Amputation of limbs due to diabetes wounds is a major concern in patients. CS-curcumin NPs of size $196.4 \mathrm{~nm}$ with a positive zeta potential of $30.3 \mathrm{mV}$ were impregnated into collagen/alginate scaffolds, and able to treat wounds at a higher rate than the control (sterile gauze) and placebo scaffolds [58]. Synergistic action of curcumin (antiinflammatory or antioxidant), CS (carrier, WH activity) and collagen (stimulant for WH activity) was persuaded.

\section{Antiobesity activity}

Obesity affects the quality of human life and is an important cause of cardiovascular, cerebrovascular, 
and metabolic diseases, which made it a major criterion in medicine and pharmacology. Alone, CS has been considered as a dietary fiber due to its indigestible, viscous nature and protection of oil droplets from digestive enzymes, especially lipase [1]. CS-insulin NPs prepared using IG method, with sizes of 296-688 nm by filtration, showed strong affinity to intestinal epithelium after $3 \mathrm{~h}$ of postoral administration and could lower the serum glucose level [59]. CS more likely acted as a cofactor for better absorption of insulin. Similarly, CS microparticles loaded with capsaicin having 600$1000 \mathrm{~nm}$ in size were prepared through the IG method for evaluation of antiobesity effects using high fat diet (HFD) in the rats [60]. Capsaicinencapsulated NPs showed a superior ability to regulate body weight, fat and serum lipids as compared to native capsaicin and commercial agent orlistat. Similar results were obtained in the treatment of HFD-induced obese rats between water insoluble and soluble CSNPs [61]. It can be inferred that CS and its derivatives, such as NPs, show an excellent capability for treatment of diabetes associated with high efficiency in drug delivery as well as effectiveness on diabetic $\mathrm{WH}$.

\section{CONCLUSION}

Chitosan nanoparticles (CSNPs) have been prepared using several methods, in which various bioactive components could be loaded for their sustained release with increased bioavailability and biological activities. Ionic gelation is the most exploited method for preparation of CSNPs. CSNPs also have the potential to enhance the absorption or availability of bioactive components by opening epithelial tight junctions and/or by direct uptake into epithelial cells via endocytosis. The augmented antimicrobial activity of CSNPs can enhance the shelf-life of various foods. Moreover, the ability of CSNPs to stabilize sensitive antioxidants, such as tea polyphenols, vitamins, etc., can help reduce the lipid or protein oxidation of foods. Hence, nanostructured forms of CS can be considered as profitable value-added products, which are used in a wide array of foods, packaging, encapsulation, biomedical remedy, and health promotion.

Acknowledgements: This work was supported by the Prince of Songkla University (AGR6302013N).

\section{REFERENCES}

1. Singh A, Benjakul S, Prodpran T (2019) Ultrasoundassisted extraction of chitosan from squid pen: molecular characterization and fat binding capacity. J Food Sci 84, 224-234.

2. Mittal A, Singh A, Benjakul S, Prodpran T, Nilsuwan K, Huda N, Caba KDL, (2020) Composite films based on chitosan and epigallocatechin gallate grafted chitosan: Characterization, antioxidant and antimicrobial activities. Food Hydrocol 111, ID 106384.

3. Singh A, Benjakul S, Prodpran T (2019) Chitooligosaccharides from squid pen prepared using different enzymes: characteristics and the effect on quality of surimi gel during refrigerated storage. Food Prod Process Nutri 1, 1-10.

4. Singh A, Mittal A, Benjakul S (2020) Full utilization of squid meat and its processing by-products: Revisit. Food Rev Int, ID 1734611.

5. Gupta A, Eral HB, Hatton TA, Doyle PS (2016) Nanoemulsions: formation, properties and applications. Soft Matter 12, 2826-2841.

6. Divya K, Jisha MS (2018) Chitosan nanoparticles preparation and applications. Environ Chem Lett 16, 101-112.

7. Agnihotri SA, Mallikarjuna N, Aminabhavi T (2004) Recent advances on chitosan-based micro-and nanoparticles in drug delivery. J Cont Rel 100, 5-28.

8. Lee JS, Kim GH, Lee HG (2010) Characteristics and antioxidant activity of Elsholtzia splendens extract-loaded nanoparticles. J Agric Food Chem 58, 3316-3321.

9. Madureira AR, Pereira A, Castro PM, Pintado $M$ (2015) Production of antimicrobial chitosan nanoparticles against food pathogens. J Food Eng 167, 210-216.

10. Zhao LM, Shi LE, Zhang ZL, Chen JM, Shi DD, Yang J, Tang ZX (2011) Preparation and application of chitosan nanoparticles and nanofibers. Braz J Chem Eng 28, 353-362.

11. Dudhani AR, Kosaraju SL (2010) Bioadhesive chitosan nanoparticles: Preparation and characterization. Carbohyd Polym 81, 243-251.

12. Budi S, Suliasih B, Rahmawati I, Erdawati (2020) Size-controlled chitosan nanoparticles prepared using ionotropic gelation. ScienceAsia 46, 457-461.

13. Debnath S, Kumar RS, Babu MN (2011) Ionotropic gelation - a novel method to prepare chitosan nanoparticles. Res J Pharm Tech 4, 492-495.

14. Kunjachan S, Jose S, Lammers T (2014) Understanding the mechanism of ionic gelation for synthesis of chitosan nanoparticles using qualitative techniques. Asian J Pharm 4, 148-153.

15. Hu B, Pan C, Sun Y, Hou Z, Ye H, Zeng X (2008) Optimization of fabrication parameters to produce chitosan-tripolyphosphate nanoparticles for delivery of tea catechins. J Agric Food Chem 56, 7451-7458.

16. Fang ZX, Bhandari B (2010) Encapsulation of polyphenols - a review. Trends Food Sci Technol 21, 510-523. 
17. Homhuan A, Harashima H, Yano I (2008) Cellular attachment and internalization of cationic liposomes containing mycobacterial cell wall. ScienceAsia 34, 179-185.

18. Nguyen TX, Huang L, Liu L, Abdalla AME, Gauthier M, Yang G (2014) Chitosan-coated nano-liposomes for the oral delivery of berberine hydrochloride. $J$ Mater Chem B 2, 7149-7159.

19. Mozafari M (2010) Nanoliposomes: preparation and analysis. In: Weissig (ed) Liposomes: Methods in Molecular Biology (Methods and Protocols), Humana Press, USA, pp 29-50.

20. Hao JP, Guo B, Yu SX, Zhang WT, Zhang DH, Wang JL, Wang Y (2017) Encapsulation of the flavonoid quercetin with chitosan-coated nano-liposomes. LWT Food Sci Technol 85, 37-44.

21. Liu N, Park HJ (2010) Factors effect on the loading efficiency of vitamin $C$ loaded chitosan-coated nanoliposomes. Colloid Surf B Biointerfaces 76, 16-19.

22. Akbari-Alavijeh S, Shaddel R, Jafari SM (2020) Encapsulation of food bioactives and nutraceuticals by various chitosan-based nanocarriers. Food Hydrocol 105, ID 105774.

23. Faikrua A, Jeenapongsa R, Sila-asna M, Viyoch J (2009) Properties of $\beta$-glycerol phosphate/collagen/chitosan blend scaffolds for application in skin tissue engineering. ScienceAsia 35, 247-254.

24. Grenha A (2012) Chitosan nanoparticles: a survey of preparation methods. J Drug Target 20, 291-300.

25. Ohya Y, Shiratani M, Kobayashi H, Ouchi T (1994) Release behavior of 5-fluorouracil from chitosan-gel nanospheres immobilizing 5-fluorouracil coated with polysaccharides and their cell specific cytotoxicity. $J$ Macromol Sci A 31, 629-642.

26. Perera U, Rajapakse N (2014) Chitosan nanoparticles: Preparation, characterization, and applications. In: Kim SK (ed) Seafood Processing By-products, Springer, USA, pp 371-387.

27. Tan C, Arshadi M, Lee MC, Godec M, Azizi M, Yan B, Eskandarloo H, Deisenroth TW, et al (2019) A robust aqueous core-shell-shell coconut-like nanostructure for stimuli-responsive delivery of hydrophilic cargo. ACS Nano 13, 9016-9027.

28. Dubey S, Mody N, Sharma R, Agrawal U, Vyas SP (2016) Nanobiomaterials: Novel nanoplatforms for protein and peptide delivery. In: Grumezescu AM (ed) Nanobiomaterials in Drug Delivery, William Andrew Publishing, USA, pp 111-146.

29. de Kruif CG, Weinbreck F, de Vries R (2004) Complex coacervation of proteins and anionic polysaccharides. Curr Opin Colloid Interface Sci 9, 340-349.

30. Butstraen C, Salaün F (2014) Preparation of microcapsules by complex coacervation of gum Arabic and chitosan. Carbohyd Polym 99, 608-616.

31. Min KA, Cho JH, Song YK, Kim CK (2016) Iron casein succinylate-chitosan coacervate for the liquid oral delivery of iron with bioavailability and stability enhancement. Arch Pharm Res 39, 94-102.

32. Augustin M, Oliver C (2012) An overview of the development and applications of nanoscale materials in the food industry. In: Huang Q (ed) Nanotechnology in the Food, Beverage and Nutraceutical Industries, Elsevier, USA, pp 3-39.

33. Augustin MA, Sanguansri P (2009) Nanostructured materials in the food industry. Adv Food Nutr Res 58, 183-213.

34. Assadpour E, Jafari SM (2019) Advances in spraydrying encapsulation of food bioactive ingredients: From microcapsules to nanocapsules. Annu Rev Food Sci Technol 10, 103-131.

35. Jafari SM, McClements DJ (2017) Nanotechnology approaches for increasing nutrient bioavailability. In: Toldrá $\mathrm{F}$ (ed) Advances in Food and Nutrition Research, Elsevier, USA, pp 1-30.

36. Haider J, Majeed H, Williams PA, Safdar W, Zhong F (2017) Formation of chitosan nanoparticles to encapsulate krill oil (Euphausia superba) for application as a dietary supplement. Food Hydrocol 63, 27-34.

37. Hasheminejad N, Khodaiyan F, Safari M (2019) Improving the antifungal activity of clove essential oil encapsulated by chitosan nanoparticles. Food Chem 275, 113-122.

38. Hadidi M, Pouramin S, Adinepour F, Haghani S, Jafari SM (2020) Chitosan nanoparticles loaded with clove essential oil: Characterization, antioxidant and antibacterial activities. Carbohyd Polym 236, ID 116075.

39. Divya K, Vijayan S, George TK, Jisha M (2017) Antimicrobial properties of chitosan nanoparticles: Mode of action and factors affecting activity. Fiber Polym 18, 221-230.

40. Dziezak JD (1988) Microencapsulation and encapsulated ingredients. Food Technol 42, 136-153.

41. Zhang H, Jung J, Zhao YJ (2016) Preparation, characterization and evaluation of antibacterial activity of catechins and catechins-Zn complex loaded $\beta$ chitosan nanoparticles of different particle sizes. Carbohydr Polym 137, 82-91.

42. Sohail MF, Javed I, Hussain SZ, Sarwar S, Akhtar S, Nadhman A, Batool S, Bukhari NI, et al (2016) Folate grafted thiolated chitosan enveloped nanoliposomes with enhanced oral bioavailability and anticancer activity of docetaxel. J Mater Chem B 4, 6240-6248.

43. Tang DW, Yu SH, Ho YC, Huang BQ, Tsai GJ, Hsieh HY, Sungd HW, Mi FL (2013) Characterization of tea catechins-loaded nanoparticles prepared from chitosan and an edible polypeptide. Food Hydrocol 30, 33-41.

44. Wang $\mathrm{YH}$, Zhang R, Qin W, Dai JW, Zhang Q, Lee KJ, Liu Y (2020) Physicochemical properties of gelatin films containing tea polyphenol-loaded chitosan nanoparticles generated by electrospray. Mater Design 185, ID 108277.

45. Maghami M, Motalebi AA, Anvar SAA (2019) Influ- 
ence of chitosan nanoparticles and fennel essential oils (Foeniculum vulgare) on the shelf life of Huso huso fish fillets during the storage. Food Sci Nutr 7, 3030-3041.

46. Wang YB, Liu L, Zhou JR, Ruan XM, Lin JD, Fu LL (2015) Effect of chitosan nanoparticle coatings on the quality changes of postharvest whiteleg shrimp, Litopenaeus vannamei, during storage at $4^{\circ} \mathrm{C}$. Food Bioprocess Tech 8, 907-915.

47. Zarei M, Ramezani Z, Ein-Tavasoly S, Chadorbaf M (2015) Coating effects of orange and pomegranate peel extracts combined with chitosan nanoparticles on the quality of refrigerated silver carp fillet. $J$ Food Process Pres 39, 2180-2187.

48. Alboghbeish H, Khodanazary A (2019) The comparison of quality characteristics of refrigerated Carangoides coeruleopinnatus fillets with chitosan and nanochitosan coating. Turk J Fish Aquat Sci 19, 957-967.

49. Antoniou J, Liu F, Majeed H, Zhong F (2015) Characterization of tara gum edible films incorporated with bulk chitosan and chitosan nanoparticles: A comparative study. Food Hydrocol 44, 309-319.

50. Al-Naamani L, Dobretsov S, Dutta J (2016) Chitosanzinc oxide nanoparticle composite coating for active food packaging applications. Innov Food Sci Emerg 38, 231-237.

51. Hosseini SF, Rezaei M, Zandi M, Farahmandghavi F (2015) Fabrication of bio-nanocomposite films based on fish gelatin reinforced with chitosan nanoparticles. Food Hydrocol 44, 172-182.

52. Hajji S, Hamdi M, Boufi S, Li SM, Nasri M (2019) Suitability of chitosan nanoparticles as cryoprotectant on shelf life of restructured fish surimi during chilled storage. Cellulos 26, 6825-6847.

53. Solval KM, Rodezno LAE, Moncada M, Bankston JD, Sathivel S (2014) Evaluation of chitosan nanoparticles as a glazing material for cryogenically frozen shrimp. LWT Food Sci Technol 57, 172-180.

54. Liang J, Cao L, Zhang L, Wan X-C (2014) Preparation, characterization, and in vitro antitumor activity of folate conjugated chitosan coated EGCG nanoparticles. Food Sci Biotechnol 23, 569-575.

55. Liu B, Wang Y, Yu Q, Li D, Li F (2018) Synthesis, characterization of catechin-loaded folateconjugated chitosan nanoparticles and their antiproliferative effect. CyTA J Food 16, 868-876.

56. Ahmed S, Ikram S (2016) Chitosan based scaffolds and their applications in wound healing. Achiev Life Sci 10, 27-37.

57. Wang T, Zheng Y, Shen Y, Shi Y, Li F, Su C, Zhao L (2018) Chitosan nanoparticles loaded hydrogels promote skin wound healing through the modulation of reactive oxygen species. Artif Cells Nanomed Biotechnol 46, 138-149.
58. Karri VVSR, Kuppusamy G, Talluri SV, Mannemala SS, Kollipara R, Wadhwani AD, Mulukutla S, Raju KRS, et al (2016) Curcumin loaded chitosan nanoparticles impregnated into collagen-alginate scaffolds for diabetic wound healing. Int J Biol Macromol 93, 1519-1529.

59. Ma Z, Lim TM, Lim LY (2005) Pharmacological activity of peroral chitosan-insulin nanoparticles in diabetic rats. Int J Pharmaceut 293, 271-280.

60. Tan SR, Gao B, Tao Y, Guo J, Su ZQ (2014) Antiobese effects of capsaicin-chitosan microsphere (CCMS) in obese rats induced by high fat diet. J Agric Food Chem 62, 1866-1874.

61. Zhang HL, Zhong XB, Tao Y, Wu SH, Su ZQ (2012) Effects of chitosan and water-soluble chitosan microand nanoparticles in obese rats fed a high-fat diet. Int J Nanomed 7, 4069-4076.

62. Keawchaoon L, Yoksan R (2011) Preparation, characterization and in vitro release study of carvacrolloaded chitosan nanoparticles. Colloid Surf B Biointerfaces 84, 163-171.

63. Ali MJM, Al-Musawi S, Pirestani M, Mahdi FR, Kazem A, Hajar R, Zuhair MH, Mahdi K, et al (2014) Curcumin-loaded chitosan tripolyphosphate nanoparticles as a safe, natural and effective antibiotic inhibits the infection of Staphylococcus aureus and Pseudomonas aeruginosa in vivo. Iran J Biotechnol 12, 1-8.

64. Arulmozhi V, Pandian K, Mirunalini S (2013) Ellagic acid encapsulated chitosan nanoparticles for drug delivery system in human oral cancer cell line (KB). Colloid Surf B Biointerfaces 110, 313-320.

65. Lamarra J, Giannuzzi L, Rivero S, Pinotti A (2017) Assembly of chitosan support matrix with gallic acidfunctionalized nanoparticles. Mat Sci Eng C Mater 79, 848-859.

66. Dube A, Nicolazzo JA, Larson I (2010) Chitosan nanoparticles enhance the intestinal absorption of the green tea catechins (+)-catechin and (-)-epigallocatechin gallate. Eur J Pharm Sci 41, 219-225.

67. Matos BN, Pereira MN, Bravo MD, Cunha M, Saldanha-Araujo F, Gratieri T, Gelfuso GM (2020) Chitosan nanoparticles loading oxaliplatin as a mucoadhesive topical treatment of oral tumors: Iontophoresis further enhances drug delivery ex vivo. Int J Biol Macromol 154, 1265-1275.

68. Unsoy G, Khodadust R, Yalcin S, Mutlu P, Gunduz U (2014) Synthesis of Doxorubicin loaded magnetic chitosan nanoparticles for $\mathrm{pH}$ responsive targeted drug delivery. Eur J Pharm Sci 62, 243-250.

69. Zhang H, Zhao Y (2015) Preparation, characterization and evaluation of tea polyphenol-Zn complex loaded $\beta$-chitosan nanoparticles. Food Hydrocol 48, 260-273. 